\title{
Moscas ectoparasitas (Diptera, Streblidae) de morcegos (Mammalia, Chiroptera) em um remanescente periurbano de Cerrado: composição da comunidade, prevalência, intensidade de infestação e especificidade
}

\author{
Jaire M. Torres ${ }^{1}$ (D), Gustavo L. Urbieta ${ }^{2}$ (D), Luciano B. M. de Almeida ${ }^{3}$ (D), \\ Driele K. F. Soares ${ }^{2}$ (i) \& Elaine A. C. dos Anjos 4 (1)
}

\begin{abstract}
1. Universidade Católica Dom Bosco, Av. Tamandaré, 6000, 79117-700 Campo Grande, MS, Brasil. (jairemarinho@gmail.com)
2. Programa de Pós-Graduação em Ciências Biológicas (Zoologia), Universidade Federal da Paraíba, João Pessoal, PB, Brasil.

3. Universidade Federal da Grande Dourados, Cidade Universitária, 79804-970 Dourados, MS, Brasil.

4. University of Manitoba, Winnipeg, Canada. (Bolsista CAPES - Processo Número 1218-13-1)
\end{abstract}

Recebido 23 março 2018

Aceito 22 janeiro 2019

Publicado 25 February 2019

$10.1590 / 1678-4766 e 2019006$

RESUMO. Moscas Streblidae ocorrem exclusivamente em morcegos e estão mundialmente distribuídas, com uma alta riqueza de moscas e hospedeiros no Brasil. Entretanto, o conhecimento dos aspectos ecológicos dessa relação é limitado à descrição das associações. O objetivo nesse trabalho foi caracterizar a comunidade de moscas ectoparasitas e de seus possíveis padrões de associação em uma área periurbana de Cerrado. Os morcegos foram capturados entre fevereiro e julho de 2011, com um esforço amostral de 9.504 h.m². Os ectoparasitas foram coletados e identificados. A relação parasito-hospedeiro foi determinada usando índices como prevalência, intensidade média de infestação e especificidade. Capturamos 161 morcegos de sete espécies, dos quais $29,81 \%(n=48)$ apresentaram-se infestados. Encontramos 83 moscas de seis espécies de Streblidae, com duas associações acidentais e oito associações não acidentais. A maior prevalência e intensidade de infestação foram verificadas na associação entre Carollia perspicillata (Linnaeus, 1758) e Trichobius joblingi Wenzel, 1966. Artibeus planirostris (Spix, 1823) também apresentou elevadas taxas de prevalência, enquanto Artibeus lituratus (Olfers, 1818) e Sturnira lilium (E, Geoffroy, 1810) revelaram baixas prevalências de infestação. Quatro espécies de Streblidae foram consideradas monoxênicas e duas oligoxênicas, o que é indicado pela análise de especificidade, o que demonstra o predomínio de espécies monoxênicas já relatado em outros trabalhos.

PALAVRAS-CHAVE. Hospedeiro, monoxênico, parasitismo, Phyllostomidae.

\begin{abstract}
Ectoparasitic flies (Diptera, Streblidae) of bats (Mammalia, Chiroptera) in a periurban remnant of Cerrado: community composition, prevalence, infestation intensity and specificity. Streblidae flies are found exclusively on bats and are distributed throughout the world, with a high richness of flies and host in Brazil. However, knowledge about the ecological aspects of these relationships is limited to descriptions of the associations. The aim of this work was to characterize the community of ectoparasites flies and their possible association patterns in a peri-urban area of Cerrado. Bats were captured between February and July 2011, with a sample effort of 9504 h.m². Ectoparasites were collected and identified. The parasite-host relationship was determined using index such as prevalence, average infestation intensity, and specificity. We captured 161 bats of seven species, with $29.81 \%(\mathrm{n}=48)$ that were infested. We found 83 flies of six species of Streblidae, with two accidental associations and eight non-accidental associations. The highest prevalence and intensity of infestation were found for the association between Carollia perspicillata (Linnaeus, 1758) and Trichobius joblingi Wenzel, 1966. Artibeus planirostris (Spix, 1823) also presented high prevalence rates, while Artibeus lituratus (Olfers, 1818) and Sturnira lilium (E. Geoffroy, 1810) had low infestation prevalence. Four species of Streblidae were considered monoxenic and two oligoxenic, which is indicated by the analysis of specificity, which demonstrates the predominance of monoxenic species already reported in other works.
\end{abstract}

KEYWORDS. Host, monoxenic, parasitism, Phyllostomidae.

Os morcegos apresentam uma elevada diversidade de artrópodes ectoparasitas, com representantes de cinco ordens, embora não sejam necessariamente restritos a esses hospedeiros (WHITAKER, 1988). Dentre os grupos associados aos morcegos, as moscas da família Streblidae se destacam por sua relação íntima com hospedeiros quirópteros, sendo ectoparasitas hematófagos encontrados exclusivamente em morcegos (Wenzel et al., 1966; Wenzel, 1976; Dick \& Patterson, 2006; PAtTerson et al., 2007). As moscas desta família apresentem-se distribuídas mundialmente (WENZEL et al., 1966; WenZEL, 1976), sendo que a maioria das espécies é encontrada na região tropical e subtropical (WHITAKER, 1988). No Brasil, são conhecidas atualmente 83 espécies e 23 gêneros de Streblidae (Graciolli, 2018).

A riqueza de moscas ectoparasitas está provavelmente relacionada à grande riqueza de hospedeiros devido ao elevado grau de especificidade parasitária (WENZEL et al., 1966). Dentre as 288 espécies encontradas na Região 
Neotropical (MicKLEBURGH et al., 2002), cerca de 184 são confirmadas no Brasil (NogueIRA et al., 2014; FeIJó et al., 2015; Morarelli \& DiAs et al., 2015; Rocha et al., 2016; GREGORIN et al., 2016; PAVAN et al., 2018), o que representa mais de $60 \%$ da riqueza de toda a região. Grande parte das espécies de moscas ectoparasitas foi reportada recentemente, o que reflete um elevado potencial para descrever novos táxons e associações parasitárias (BARBIER \& GRACIOLLI, 2016). Entretanto, a maioria dos estudos realizados com essas moscas é de caráter descritivo ou quantitativo, limitando a compreensão sobre sua biologia e coevolução com morcegos.

Poucos estudos avaliam as associações entre moscas e morcegos em regiões de Cerrado utilizando índices parasitológicos como a prevalência, intensidade de infestação em uma espécie de hospedeiro ou mesmo a especificidade entre as associações (e.g. Aguiar \& ANTONINI, 2011; ERIKSSON et al., 2011; URBIETA et al., 2014; VASCONCELOS et al., 2016; AgUIAR \& ANTONINI, 2016; BARBIER \& GRACIOLLI, 2016; DoRNELles et al., 2017). Esses parâmetros preenchem lacunas sobre a ecologia do parasitismo, e podem ser aplicados para avaliar fatores que influenciam nas associações, como o clima (Lourenço \& PaLmerim, 2007) ou os aspectos reprodutivos dos hospedeiros (BERTOLA et al., 2005).

A falta de estudos com a aplicação de índices parasitológicos padronizados para as associações entre moscas ectoparasitas e morcegos é um fator limitante para a identificação e determinação de padrões, impedindo a comparação com outras regiões melhor estudadas (BARBIER \& Graciolli, 2016). Os índices parasitológicos podem proporcionar o primeiro passo para a compreensão da intensidade da relação entre estes grupos, além de fornecer subsídios essenciais para inferir questões relacionadas aos mecanismos evolutivos dessas associações (VASCONCELOS et al., 2016).

O conhecimento das associações entre as moscas ectoparasitas e morcegos apresenta lacunas evidentes nas regiões de Cerrado, embora seja esperada alta especificidade parasita-hospedeiro (BARBIER \& Graciolli, 2016). Objetivou-se descrever a comunidade de moscas Streblidae em morcegos de uma área periurbana de Cerrado, buscando possíveis padrões de associação por meio de índices parasitológicos.

\section{MATERIAL E MÉTODOS}

Área de estudo. As capturas foram realizadas na Fazenda Cervinho (1957'18.36”'S, 54²0'8.80”O), nos limites do município de Bandeirantes, Mato Grosso do Sul. O clima da região é classificado como Tropical Chuvoso de Savana (Aw), segundo a classificação de Köppen (PeEL et al., 2007). Essa região apresenta duas estações bem definidas, com um inverno seco e um verão chuvoso, onde a temperatura média é $36^{\circ} \mathrm{C}$ e a precipitação média anual é de $1,532 \mathrm{~mm}$.

A fazenda se localiza a cerca de cinco quilômetros de distância do perímetro urbano do município, a uma altitude média de 630 a.n.m. A área total é de 900 hectares, dos quais cerca de 60 são compostos por sua Reserva Legal (RL) e
Área de Proteção Permanente (APP), onde são encontradas porções de cerradão, cerrado strictu sensu, mata ciliar e vereda. Além desses ambientes, é encontrada ainda uma porção de cerradão em processo de regeneração natural, onde foram interrompidos processos históricos de cortes seletivos e da utilização para manejo de gado por um período de dez anos antes do desenvolvimento do estudo.

Captura de morcegos e coleta de ectoparasitas. Foram realizadas duas capturas mensais em noites consecutivas entre fevereiro e julho de 2011. Foram estabelecidos quatro pontos de captura na área: 1) mata ciliar; 2) cerradão, 3) cerrado strictu sensu e 4) área de regeneração natural. A amostragem de cada ponto ocorreu em sistema de rodízio, com sorteio da sequência de locais amostrados.

Para a coleta dos animais foram utilizadas seis redes de neblina, três com dimensões de $12,0 \times 2,5 \mathrm{~m}$ e três com $7,0 \times 2,0 \mathrm{~m}$, que permaneceram abertas pelo período de seis horas por noite e foram vistoriadas em intervalos de 30 minutos. O esforço amostral empregado foi de 2.376 h.m ${ }^{2}$ em cada ponto de coleta, totalizando 9.504 h.m. ${ }^{2}$ de esforço em toda a extensão da área, calculado segundo STRAUBE \& BIANCONI (2002).

Os morcegos capturados foram triados em campo e identificados segundo as diagnoses constantes em REIS et al. (2007) e GREGORIN \& TADDEI (2002). As moscas ectoparasitas foram coletadas com o auxílio de pinça de ponta fina e pincéis umedecidos com álcool, e fixados em álcool etílico $70 \%$ em recipientes individuais para cada hospedeiro. Em laboratório, as moscas foram identificadas com a ajuda de um estereomicroscópio de acordo com GuERREIRO (1993, 1994a,b, 1995a,b) e Graciolli \& CARVALHO (2001).

Espécimes de morcegos não identificados em campo e espécimes testemunhos foram coletados e depositados na coleção zoológica da Universidade Católica Dom Bosco (UCDB) com os códigos LZV 021 à LZV025, LZV 033 à LZV 039 e LZV 042 à LZV 045. Após identificação, as moscas foram depositadas na coleção entomológica da mesma instituição sob os códigos CEM 001 à CEM 054. Todos os procedimentos foram conduzidos mediante a licença ambiental Sisbio 21548-2.

Análise dos dados. As comunidades de ectoparasitas e os índices de parasitismo foram determinados para cada espécie de hospedeiro. Para isso, foram calculadas a prevalência $(\mathrm{P}=$ número de indivíduos parasitados/número de indivíduos examinados) e a intensidade média de infestação (IM = número de parasitos/número de hospedeiros infestados) para cada associação parasito-hospedeiro segundo BusH et al. (1997), permitindo inferir a intensidade das associações entre as moscas ectoparasitas e os morcegos.

As associações parasito-hospedeiro foram estabelecidas utilizando-se ainda o índice de especificidade $\left(S_{\mathrm{TD}}{ }^{*}\right)$ proposto por Poulin \& Mouillot (2005), que inclui informações taxonômicas e ecológicas. O índice $S_{\mathrm{TD}}$ * mede as distâncias taxonômicas de todas as espécies hospedeiras usadas por um determinado parasita e atribui um peso para cada nível taxonômico (VASCONCELOS et al., 2016). O valor do $S_{\text {TD }}{ }^{*}$ varia de 0 a 5 , permitindo a classificação dos parasitas 
como: I) Monoxênicos: apresentam valor igual a 0, indicando uma única espécie hospedeira; II) Oligoxênicos: apresentam valor igual a 1, indicando hospedeiros congêneres; ou III) Não específicos: apresentam valor maior que 1, diretamente proporcional a quantidade de hospedeiros possíveis. Para a realização da avaliação de especificidade foram excluídas associações acidentais de acordo com GRACIOLLI et al. (2008). Para a inferência das distâncias taxonômicas das espécies de morcegos foi utilizada a classificação taxonômica compilada por REIS et al. (2007).

Os cálculos de prevalência e intensidade média de infestação foram executados no software Quantitative Parasitology 3.0 (RózsA et al., 2000), assim como os intervalos de confiança com 2.000 randomizações. A determinação $S_{\mathrm{TD}}$ * foi realizada no programa TaxoBiodiv 1.2 (www.otago.ac.nz/parasitegroup/downloads.html).

\section{RESULTADOS}

Foram capturados e examinados 161 morcegos, com representantes de seis espécies de Phyllostomidae $(\mathrm{n}=160)$ e uma única de Molossidae $(\mathrm{n}=1)$ (Tab. I). As espécies mais abundantes foram Carollia perspicillata (Linnaeus, 1758) e Artibeus planirostris (Spix, 1823), representando aproximadamente $22,98 \%(\mathrm{n}=37)$ e $22,34 \%(\mathrm{n}=36)$ das capturas, respectivamente (Tab. I).

Dentre os morcegos capturados, somente 29,81\% $(\mathrm{n}=48)$, apresentavam-se parasitados por moscas, sendo todos eles filostomídeos. Foram coletadas ao todo 83 moscas de seis espécies, todas pertencentes à Streblidae, sendo as mais abundantes Trichobius joblingi Wenzel, $1966(\mathrm{n}=35)$, Megistopoda aranea (Coquillet, 1899) $(\mathrm{n}=22)$ e Aspidoptera phyllostomatis (Perty, 1833) $(\mathrm{n}=13)$.

Tab. I. Moscas ectoparasitas de morcegos (Diptera: Streblidae) e seus hospedeiros coletados na Fazenda Cervinho, Bandeirantes, Mato Grosso do Sul, Brasil (N, número de espécimes; HI, hospedeiros infestados; P, prevalência de infestação; IM, Intensidade Média de Infestação; S *, índice de especificidade; a , associações acidentais; ${ }^{\text {b }}$, dados insuficientes para cálculo de intervalo de confiança).

\begin{tabular}{|c|c|c|c|c|c|}
\hline $\begin{array}{l}\text { FAMÍLIA DE MORCEGO } \\
\text { Espécie de morcego } \\
\text { Espécie de mosca }\end{array}$ & $\mathrm{N}$ & $\mathrm{HI}$ & $\mathrm{P}$ & IM & $\mathrm{S}_{\mathrm{TD}}^{*}$ \\
\hline \multicolumn{6}{|l|}{ PHYLLOSTOMIDAE } \\
\hline Artibeus planirostris (Spix, 1823) & 36 & & & & \\
\hline Megistopoda aranea (Coquillet, 1899) & 22 & 14 & $\begin{array}{c}38,9 \\
(0,23-0,56)\end{array}$ & $\begin{array}{c}1,57 \\
(1,21-1,86)\end{array}$ & 1,00 \\
\hline Aspidoptera phyllostomatis (Perty, 1833) & 13 & 8 & $\begin{array}{c}22,2 \\
(0,10-0,39)\end{array}$ & $\begin{array}{c}1,63 \\
(1,13-2,25)\end{array}$ & 1,00 \\
\hline Aspidoptera falcata Wenzel, $1976(1)^{\mathrm{a}}$ & 1 & 1 & $\begin{array}{c}2,8 \\
(0,00-0,14)\end{array}$ & $1,00^{\mathrm{b}}$ & - \\
\hline Megistopoda proxima (Séguy, 1926) ${ }^{\mathrm{a}}$ & 1 & 1 & $\begin{array}{c}2,8 \\
(0,00-0,14)\end{array}$ & $1,00^{\mathrm{b}}$ & - \\
\hline Artibeus lituratus (Olfers, 1818) & 29 & & & & \\
\hline Megistopoda aranea (Coquillet, 1899) & 2 & 2 & $\begin{array}{c}6,9 \\
(0,00-0,22)\end{array}$ & $1,00^{\mathrm{b}}$ & 1,00 \\
\hline Paratrichobius longicrus (Miranda Ribeiro, 1907) & 1 & 1 & $\begin{array}{c}3,4 \\
(0,00-0,17)\end{array}$ & $1,00^{\mathrm{b}}$ & 0,00 \\
\hline Aspidoptera phyllostomatis (Perty, 1833) & 1 & 1 & $\begin{array}{c}3,4 \\
(0,00-0,17)\end{array}$ & $1,00^{\mathrm{b}}$ & 1,00 \\
\hline Carollia perspicillata (Linnaeus, 1758) & 37 & & & & \\
\hline Trichobius joblingi Wenzel, 1966 & 11 & 15 & $\begin{array}{c}40,5 \\
(0,24-0,57)\end{array}$ & $\begin{array}{c}2,33 \\
(1,60-3,13)\end{array}$ & 0,00 \\
\hline Sturnira lilium (E. Geoffroy, 1810) & 22 & & & & \\
\hline Megistopoda proxima (Séguy, 1926) & 3 & 2 & $\begin{array}{c}9,1 \\
(0,01-0,29)\end{array}$ & $\begin{array}{c}1,50 \\
(1,00-1,50)\end{array}$ & 0,00 \\
\hline Aspidoptera falcata Wenzel, 1976 & 3 & 3 & $\begin{array}{c}13,6 \\
(0,02-0,34)\end{array}$ & $\begin{array}{c}1,33 \\
(1,00-1,67)\end{array}$ & 0,00 \\
\hline Platyrrhinus lineatus (E. Geoffroy, 1810) & 24 & 0 & - & - & - \\
\hline Glossophaga soricina (Pallas, 1766) & 12 & 0 & - & - & - \\
\hline \multicolumn{6}{|l|}{ MOLOSSIDAE } \\
\hline Molossops teminckii (Burmeister, 1854) & 1 & 0 & - & - & - \\
\hline Total & 244 & 48 & & & \\
\hline Morcegos & 161 & & & & \\
\hline Moscas & 83 & & & & \\
\hline
\end{tabular}


As maiores prevalências e intensidades média de infestação foram registradas nas associações entre C. perspicillata com T. joblingi $(40,5 \% ; 2,33)$, Artibeus planirostris com $M$. aranea $(38,9 \% ; 1,57)$ e $A$. planirostris com A. phyllostomatis $(22,2 \% ; 1,63)$. Foram encontradas somente duas associações acidentais, Aspidoptera falcata Wenzel, 1976 e Megistopoda proxima (Séguy, 1926) em A. planirostris. Todas as outras associações verificadas são conhecidas e consideradas não acidentais.

Em termos de especificidade, aproximadamente 67\% das espécies de moscas avaliadas estiveram associadas a um único hospedeiro e foram consideradas monoxênicas $\left(S_{\mathrm{TD}}{ }^{*}=0\right)$. Megistopoda aranea e $A$. phyllostomatis foram as únicas espécies oligoxênicas registradas em associação com A. planirostris e Artibeus lituratus (Olfers, 1818) $\left(S_{\mathrm{TD}}^{*}=1\right)$ (Tab. I).

\section{DISCUSSĀO}

A composição de moscas verificada em cada espécie de hospedeiro apresentou um padrão esperado, seguindo as associações apontadas por GRACIOLLI et al. (2008), verificandose associações acidentais somente entre $A$. planirostris e moscas $A$. falcata e $M$. proxima. Porém, quando avaliados os índices parasitológicos, são observadas algumas variações quanto à prevalência e intensidade de infestação relatadas.

A associação entre Carollia perspicillata e T. joblingi é recorrente em diversos estudos realizados no Cerrado (GRACIOLli et al., 2006a, b; ERIKSSON et al., 2011; SANTOS et al., 2013; VASCONCELOS et al., 2016; BARBIER \& GRACIOLLI, 2016, DoRnelles et al. 2017; Graciolli et al. 2017). Os estudos realizados no Cerrado não apresentam um padrão definido quanto aos valores de infestação dessa relação, com prevalência variando de 40,5\% (ERIKSON et al. 2011) a 91\% (Aguiar \& ANTONINI, 2016) e intensidade média de infestação variando de 2,3 (SANTOS et al., 2013) a 3,5 (BARBIER $\&$ Graciolli, 2016). Entretanto, os poucos estudos que descrevem os índices de parasitismo nessas regiões (ERIKSON et al., 2011; SANTOS et al., 2013; VASCONCELOS et al., 2016; Aguiar \& ANTONini, 2016; Barbier \& GRaCiolli, 2016; DORNELles et al., 2017) demonstraram uma tendência a infestações mais intensas em populações de $C$. perspicillata com maiores prevalências de parasitismo.

Artibeus planirostris apresentou a maior riqueza de moscas, parasitada principalmente por M. aranea, sendo essa uma associação usual (GUERRERo, 1994a,b) e amplamente registrada em regiões de Cerrado (GraCIOLLI et al., 2006 a,b; Graciolli et al., 2010; SANTOS et al., 2013; VASCONCELOS et al., 2016), inclusive na região do Mato Grosso do Sul (ERIKSSON et al., 2011; URBIETA et al., 2014; BARBIER \& GraCiOlli, 2016; Dornelles et al., 2017; GRACIOlli et al. 2017). A prevalência e a intensidade média de infestação de M. aranea e A. phyllostomatis em A. planirostris pode ser considerada elevada, pois foi superior às relatadas em outros estudos realizados no Cerrado (e.g. ERIKSsON et al., 2011; SANTOS et al., 2013; URBIETA et al., 2014; VASCONCELOS et al., 2016; BARBIER \& GRACIOLLI, 2016).
Embora apresentem várias espécies de moscas associadas, indivíduos de A. lituratus demonstraram baixos valores de prevalência de infestação para as três espécies de moscas encontradas. Essa espécie de morcego é frequentemente relatada com baixas prevalências de infestação em diversos estudos no Brasil (e.g. CAmilloti et al., 2010; FrAnÇA et al., 2013; LOURENÇO et al., 2014; URBIETA et al., 2014; SoARes et al., 2016; Dornelles et al., 2017), podendo chegar à ausência de parasitismo (e.g. ERIKSON et al., 2011; BARBIER \& GRACIOLLI, 2016), e com maiores prevalências relatadas em sua associação com $A$. phyllostomatis (RUI \& GRACIOLLI, 2005; FrANÇA et al., 2013). Quando comparado aos índices já descritos no Cerrado, o parasitismo por Paratrichobius longicrus (Miranda Ribeiro, 1907) apresentou menor prevalência e similar intensidade média de infestação do que o já descrito por VASCONCELOS et al. (2015), enquanto o parasitismo verificado por M. aranea demonstrou menor prevalência e maior intensidade média de infestação do que os relatados por URBIETA et al. (2014) em remanescentes urbanos de Cerrado.

A ocorrência de M. proxima e A. falcata em S. lilium já foi descrita no Cerrado do Mato Grosso do Sul (Graciolli et al., 2006a, b; ERIKSSON et al., 2011; BARBIER \& GRACIOLLI, 2016; DorNelles et al., 2017; GRACIOLLI et al. 2017), porém em prevalências maiores do que as relatadas aqui. Quanto à intensidade média de infestação, ambas as espécies de moscas apresentaram associações de menor ou similar intensidade do que as verificadas em outras regiões do Mato Grosso do Sul (ERIKSSON et al., 2011; BARBIER \& GRACIOLLI, 2016).

Os valores das associações utilizando o $S_{\mathrm{TD}}{ }^{*}$ indicaram que $67 \%$ das espécies de moscas encontradas são monoxênicas, enquanto $33 \%$ das espécies parasitaram espécies congêneres, sendo consideradas oligoxênicas. A representatividade na riqueza de espécies monoxênicas apresentou-se abaixo do padrão conhecido, que é relatado variando de $71 \%$ a $88 \%$ em diversos estudos (e.g. WenzeL et al., 1966; Marshall, 1981; Dick \& Patterson, 2007; VASCONCELOS et al., 2016), indicando menor especificidade nas comunidades de moscas estudadas.

A especificidade é amplamente assumida para as relações entre moscas ectoparasitas e morcegos, mas somente dois trabalhos utilizando o $S_{\mathrm{TD}}$ * no Brasil foram desenvolvidos até o momento: VASCONCELOS et al. (2016) que registraram 76\% de espécies monoxênicas em Matas Secas de Cerrado e URBIETA et al. (in press) que registraram 71\% de espécies monoxênicas em áreas urbanas de Cerrado. A diferença (4\%) entre a o valor registrado no presente trabalho do menor valor comumente relatado $(71 \%)$ possivelmente seja efeito indireto do baixo número de morcegos e moscas capturados em relação aos dois trabalhos.

As espécies de moscas de Streblidae costumam apresentar especificidade elevada, o que é geralmente explicado por uma baixa capacidade de sobrevivência em hospedeiros não habituais (FrITZ, 1983). O sucesso na utilização de hospedeiros inespecíficos depende da ruptura de barreiras de dispersão geralmente atuantes sobre uma espécie de parasita, como as incompatibilidades fisiológicas, 
limitações no encontro de hospedeiros e na dificuldade em se encontrar parceiros sexuais (COMBES, 1991; VASCONCELOS et al., 2016). O sucesso na ruptura dessas barreiras resulta na diminuição da especificidade de uma espécie de parasita (VASCONCElos et al., 2016), como ocorre com as espécies oligoxênicas A. phyllostomatis e M. aranea. De acordo com Dick et al. (2009), a ausência de hospedeiros primários pode levar à quebra dessas barreiras, tornando possível aos ectoparasitas colonizarem novos hospedeiros. Em contrapartida, A. phyllostomatis e $M$. aranea são comumente relatadas como parasitas primários de várias espécies de Artibeus (Graciolli \& CARVAlHo, 2001; Graciolli \& RUI, 2001; Prevedello et al., 2005), podendo sua distribuição na comunidade de morcegos estar mais relacionada ao comportamento e distribuição das espécies de Artibeus do que à especificidade.

Agradecimentos. Ao Programa Institucional de Bolsas de Iniciação Científica (PIBIC) da Universidade Católica Dom Bosco pelo financiamento da pesquisa. Ao senhor Alberto Penze Campanha pela autorização do desenvolvimento da pesquisa na área da Fazenda Cervinho. Ao Instituto Chico Mendes de Conservação da Biodiversidade pela concessão da licença número 21 548-2 para o desenvolvimento das atividades de captura de morcegos e coleta de amostras.

\section{REFERÊNCIAS BIBLIOGRÁFICAS}

Aguiar, L. M. S. \& Antonini, Y. 2011. Descriptive ecology of bat flies (Diptera: Hippoboscoidea) associated with vampire bats (Chiroptera: Phyllostomidae) in the cerrado of central Brazil. Memórias do Instituto Oswaldo Cruz 106(2): 170-176.

Aguiar, L. M. S. \& Antonini, Y. 2016. Prevalence and intensity of Streblidae in bats from a Neotropical savanna region in Brazil. Folia Parasitologica 63:1-8

Barbier, E. \& Graciolli, G. 2016. Community of bat flies (Streblidae and Nycteribiidae) on bats in the Cerrado of Central- West Brazil: hosts, aggregation, prevalence, infestation intensity, and infracommunities. Studies on Neotropical Fauna and Environment 51:176-187.

Bertola, P. B.; Aires, C. C.; Favorito, S. E.; Graciolli, G.; Amaku, M. \& Rocha, M. P. 2005. Batflies (Diptera: Streblidae, Nycteribiidae) parasitic on bats (Mammalia: Chiroptera) at Parque Estadual da Cantareira, São Paulo, Brazil: parasitism rates and host-parasite associations. Memórias do Instituto Oswaldo Cruz 100(1):25-32.

Bush, A. O.; Lafferty, K. D.; Lotz, J. M. \& Shostak, A.W. 1997. Parasitology meets Ecology on its own terms: Margolis et al. revisited. Journal of Parasitology 83(4):575-583.

Camilotti, V. L.; Graciolli, G.; Weber, M. M.; Arruda, J. L. S. \& Cáceres, N. C. 2010. Bat flies from the deciduous Atlantic Forest in southern Brazil: Host-parasite relationships and parasitism rates. Acta Parasitologica 55(2):194-200.

Combes, C. 1991. Evolution of parasite life cycles. In: Toft, C. A.; Aeschlimann, A. \& Bolis, L. eds. Parasite-host associations: coexistence or conflict? Oxford, Oxford University Press, p. 62-82

Dick, C. W. \& PATTERSON, B. D. 2006. Bat flies: Obligate ectoparasites of bats. In: Morand, S.; Krasnov, B. R. \& Poulin, R. eds. Micromammals and Macroparasites: from Evolutionary Ecology to Management. Tokyo, Springer-Verlag, p. 178-194.

Dick, C. W. \& Patterson, B. D. 2007. Against all oddos: explaining high host specificity in dispersal-prone parasites. International Journal for Parasitology 37(8-9):871-876.

Dick, C. W.; Esberárd, C. E. L.; Graciolli, G.; Bergallo, H. G. \& GetTINGER, D. 2009. Assessing host specificity of obligate ectoparasitesin the absence of dispersal barriers. Parasitology Research 105:1345-1349.

Dornelles, G. D. P.; Graciolli, G.; Odon, A. \& Bordignon, M. O. 2017. Infracommunities of Streblidae and Nycteribiidae (Diptera) on bats in an ecotone area between Cerrado and Atlantic Forest in the state of Mato Grosso do Sul. Iheringia, Série Zoologia 107:1-5.

Eriksson, A.; Graciolli, G. \& Fischer, E. 2011. Bat flies on phyllostomid hosts in the Cerrado region: component community, prevalence and intensity of parasitism. Memórias do Instituto Oswaldo Cruz 106(3):274-278.

Feisó, A.; Rocha, P. A. \& Althoff, S. L. 2015. New species of Histiotus (Chiroptera: Vespertilionidae) from northeastern Brazil. Zootaxa 4048:412-427.

França, D. S.; Pereira, S. N.; MaAs, A. C. S.; Martins, M. A.; Bolzan, D. P.; Lima, I. P.; Dias, D. \& Peracchi, A. L. 2013. Ectoparasitic flies (Diptera, Streblidae) of bats (Chiroptera, Phyllostomidae) in an Atlantic Forest area, southeastern Brazil. Revista Brasileira de Zoologia 73(4):847-854.

FritZ, G. N. 1983. Biology and ecology of bat flies (Diptera: Streblidae) on bats of the genus Carollia. Journal of Medicine Entomology 20:1-10.

Graciolli, G.; Azevedo, A. A.; Árzua, M.; Barros-Battesti, D. M. \& LINARDI, P. M. 2008. Artrópodos ectoparasitos de morcegos no Brasil. In: PACheco, S.; Marques, R. V. \& EsberáRd, C. E. L. orgs. Morcegos do Brasil: Biologia, Sistemática, Ecologia e Conservação. Porto Alegre, Armazém Digital, p. 123-138.

Graciolli, G. \& Carvalho, C. J. B. 2001. Moscas ectoparasitas (Diptera, Hippoboscoidea, Nycterybiidae) de morcegos (Mammalia: Chiroptera) do Estado do Paraná, Brasil. II. Streblidae. Chave pictórica para os gêneros e espécies. Revista Brasileira de Entomologia 18:907-960.

Graciolli, G.; Carvalho, L. F. A. C.; Eriksson, A. F.; Silva, C. L. \& AlCÂntara, D. M. C. 2017. Lista das espécies de Hippoboscoidea (Diptera) no estado de Mato Grosso do Sul, Brasil. Iheringia, Série Zoologia 107:1-10.

Graciolli, G.; Cáceres, N. S. \& Bornschein, M. R. 2006b. Novos registros de moscas ectoparasitas (Diptera, Streblidae e Nycteribiidae) de morcegos (Mammalia, Chiroptera) em áreas de transição cerradofloresta estacional no Mato Grosso do Sul, Brasil. Biota Neotropica 6(2).

Graciolli, G. \& Coelho, D. C. 2001. Streblidae (Diptera, Hippoboscoidea) sobre morcegos filostomídeos (Chiroptera, Phyllostomidae) em cavernas do Distrito Federal, Brasil. Revista Brasileira de Zoologia 18:965-970.

Graciolli, G.; Passos, F. C.; Pedro, W. A. \& Lim, B. K. 2006a. Moscas ectoparasitas (Diptera, Streblidae) de morcegos filostomídeos (Mammalia, Chiroptera) na Estação Ecológica dos Caetetus, São Paulo, Brasil. Revista Brasileira de Zoologia 23(1):298-299.

Graciolli, G. \& RUI, A. M. 2001. Streblidae (Diptera, Hippoboscoidea) em morcegos (Chiroptera, Phyllostomidae) no nordeste do Rio Grande Do Sul, Brasil. Iheringia, Série Zoologia 90:85-92.

Graciolli, G.; Zortea, M. \& Carvalho, L. F. A. C. 2010. Bat flies (Diptera, Streblidae and Nycteribiidae) in a Cerrado area of state of Goias, Brazil. Revista Brasileira de Entomologia 54:511-514.

Graciolli, G. 2018. Streblidae in Catálogo Taxonômico da Fauna do Brasil. PNUD. Disponível em: $<$ http://fauna.jbrj.gov.br/fauna/ faunadobrasil/2624>. Acesso em: 17 Outubro 2018.

Gregorin, R.; Moras, L. M.; Acosta, L. H.; Vasconcellos, K. L.; Poma, J. L.; Santos, F. R. \& PaCA, R. C. 2016. A new species of Eumops (Chiroptera: Molossidae) from southeastern Brazil and Bolivia. Mammalian Biology 81:235-246.

GREGORIN, R. \& TADDEI, V. A. 2002. Chave artificial para a identificação de molossídeos brasileiros (Mammalia: Chiroptera). Mastozoología Neotropical 9(1):13-32.

Guerrero, R. 1993. Catalogo de los Streblidae (Diptera: Pupipara) parasitos de murcielagos (Mammalia: Chiroptera) dei Nuevo Mundo. I. Clave para los generos y Nycterophilinae. Acta Biologica Venezuelica 14:61-75

Guerrero, R. 1994a. Catalogo de los Streblidae (Diptera: Pupipara) parasitos de murcielagos (Mammalia: Chiroptera) dei Nuevo Mundo. II. Los grupos: pallidus, caecus, major, uniformis y longipes dei genero Trichobius Gervais, 1844. Acta Biologica Venezuelica 15:1-18

Guerrero, R. 1994b. Catalogo de los Streblidae (Diptera: Pupipara) parasitos de murcielagos (Mammalia: Chiroptera) dei Nuevo Mundo. IV. Trichobiinae com alas desarrolladas. Boletín de Entomologia Venezolana 9:161-192.

Guerrero, R. 1995a. Catalogo de los Streblidae (Diptera: Pupipara) parasitos de murcielagos (Mammalia: Chiroptera) dei Nuevo Mundo. 
III. Los grupos: dugesii, dunni y phyllostomae dei genero Trichobius Gervais, 1844. Acta Biologica Venezuelica 15:1-27.

Guerrero, R. 1995b. Catalogo de los Streblidae (Diptera: Pupipara) parasitos de murcielagos (Mammalia: Chiroptera) dei Nuevo Mundo. V. Trichobiinae con alas reducidas o ausentes y miscelaneos. Boletín de Entomologia Venezolana 10(2):135-160.

Lourenço, S. I. \& Palmerim, J. M. 2007. Can mite parasitism affect the conditions of hosts? Implications for the social structure of the colonial bats. Journal of Zoology 273:161-168.

Lourenço, E. C.; Patrício, P. M. P.; Pinheiro, M. C.; Dias, R. M. \& FAMADAS, K. M. 2014. Streblidae (Diptera) on bats (Chiroptera) in an area of Atlantic Forest, state of Rio de Janeiro. Revista Brasileira de Parasitologia Veterinária 23(2):1-7.

Marshall, A. G. 1981. Ecology of Ectoparasites Insects. London, Academic Press. $459 \mathrm{p}$

Mickleburgh, S.P.; Hutson, A. M. \& Racey, P. A. 2002. A review of the global conservation status of bats. Oryx 36(1):18-34.

MoRATELLI, R. \& Dias, D. 2015. A new species of nectar-feeding bat, genus Lonchophylla, from the Caatinga of Brazil (Chiroptera, Phyllostomidae). ZooKeys 514:73-91.

Nogueira, M. R.; Lima, I. P.; Moratelli, R.; TaVares, V. C.; Gregorin, R. \& Peracchi, A. L. 2014. Checklist of Brazilian bats, with comments on original record. Check List 10:808-821.

Pavan, C. A.; Bobrowiec, E. D. \& Percequillo, A. R. 2018. Geographic variation in a South American clade of mormoopid bats, Pteronotus (Phyllodia), with description of a new species. Journal of Mammalogy 99(3):624-645.

Patterson, B. D.; Dick, C. W. \& Dittmar, K. 2007. Roosting habits of bats affect their parasitism by bat flies (Diptera: Streblidae). Journal of Tropical Ecology 23(2):177-189.

Peel, M.C.; Finlayson, B.L. \& Macmahon, T.A. 2007. Updated world map of the Köppen-Geiger climate classification. Hydrology and Earth System Science 11:1633-1644.

Poulin, R. \& Mouillot, D. 2005. Combining phylogenetic and ecological information into a new index of host specificity. Parasite specialization from a phylogenetic perspective: a new index of host specificity. Journal Parasitology 91:511-514.

Prevedello, J. A.; Graciolli, G. \& Carvalho, C. J. B. 2005. A fauna de dípteros (Streblidae e Nycteribiidae) ectoparasitos de morcegos (Chiroptera) do Estado do Paraná, Brasil: composição, distribuição e áreas prioritárias para novos estudos. Biociências 13(2):193-209.

Reis, N. R.; PerRacChI, A. L.; Pedro, W. A. \& Lima, I. P. 2007. Morcegos do Brasil. Londrina, Editora da Universidade Estadual de Londrina. 253p.
Rocha, P. A.; Brandão, M. V. B.; Garbino, G. S. T.; Cunha, I. N. \& AIRES, C. C. 2016. First record of Salvin's big-eyed bat Chiroderma salvini Dobson, 1878 for Brazil. Mammalia 80:5.

Rózsa, L.; Reiczigel, J. \& Majoros, G. 2000. Quantifying parasites in samples of hosts. Journal of Parasitology 86(2):228-232.

RUI, A. M. \& Graciolli, G. 2005. Moscas ectoparasitas (Diptera, Streblidae) de morcegos (Chiroptera, Phyllostomidae) no sul do Brasil: associações hospedeiros-parasitos e taxas de infestação. Revista Brasileira de Zoologia 22(2):438-445.

Santos, C. L. C.; Pereira, A. C. N.; Bastos, V. J. C.; Graciolli, G. \& RebêLo, J. M. M. 2013. Parasitism of ectoparasitic flies on bats in the northern Brazilian Cerrado. Acta Parasitologica 58(2):207-214.

Soares, F. A. M.; Graciolli, G.; Ribeiro, C. E. B. P.; Bandeira, R. S.; Moreno, J. A. T. \& Ferrari, S. F. 2016. Bat (Mammalia: Chiroptera) diversity in an area of Mangrove Forest in southern Pernambuco, Brazil, with a new species record and notes on ectoparasites (Diptera: Streblidae). Papéis Avulsos de Zoologia 56(6):63-68.

Straube, F. C. \& Bianconi, G. V. 2002. Sobre a grandeza e a unidade utilizada para estimar esforço de captura com utilização de redes-deneblina. Chiroptera Neotropical 8(1-2):150-152.

Urbieta, G. L.; Torres, J. M.; Almeida, L. B. M.; Shinohara, A. \& Anjos, E. A. C. 2014. Infestação de morcegos (Mammalia, Chiroptera) por moscas do gênero Megistopoda (Diptera, Streblidae) em um fragmento urbano de Cerrado de Campo Grande, Mato Grosso do Sul. Boletim da Sociedade Brasileira de Mastozoologia 69:10-13.

Urbieta, G. L.; Torres, J. M.; Anjos, E. A. C.; Carvalho, C.M.E. \& GraCiOLli, GParasitism of bat flies (Nycteribiidae and Streblidae) on bats in urban environments: lower prevalence, infracommunities, and specificity. Acta Chiropterologica 20(2):511-518.

Vasconcelos, P. F.; Falcão, L. A. D.; Graciolli, G. \& Borges, M. A. S 2016. Parasite-host interactions of bat flies (Diptera: Hippoboscoidea) in Brazilian tropical dry forests. Parasitology Research 115(1):367-377.

Wenzel, R. L. 1976. The streblid batflies of Venezuela (Diptera: Streblidae). Brigham Young University Science Bulletin, Biological Series 20(4):1-177.

Wenzel, R. L.; Tipton, V. J. \& Kiewlicz, A. 1966. The streblid bat flies of Panama (Diptera: Calyptera: Streblidae). In: Wenzel, R. L. \& TIPTON, V. J. eds. Ectoparasites of Panama. Chicago, Field Museum of Natural History, p. 405-675.

WhitAKER JR, J. O. 1988. Collecting and preserving ectoparasites for ecological study. In: KunZ, J. H. ed. Ecological and Behavioral Methods for the Study of Bats. Washington, Smithsonian Institution Press, p. 459-474. 